\title{
Efficacy of a Novel Intranasal Formulation of Azelastine Hydrochloride and Fluticasone Propionate, Delivered in a Single Spray, for the Treatment of Seasonal Allergic Rhinitis: Results from Russia
}

\author{
Natalia I. Ilyina ${ }^{a}$ Anton S. Edin ${ }^{b}$ Natalia G. Astafieva ${ }^{c}$ Andrey S. Lopatin ${ }^{d}$ \\ Irina V. Sidorenko ${ }^{e}$ Olga P. Ukhanova ${ }^{f}$ Fatimat M. Khanovag \\ ${ }^{a}$ Federal State Budget Institution, State Scientific Center Institute of Immunology of the Federal Biomedical Agency, \\ Moscow, Russia; 'bLC Alliance Biomedical Russian Group, Saint Petersburg, Russia; ' $S t a t e$ Budget Educational Institution of \\ Higher Professional Education, Saratov State Medical University named after V.I. Razumovsky of the Ministry of Healthcare of \\ the Russian Federation, Saratov, Russia; ${ }^{\mathrm{d}}$ Federal State Institution Polyclinic N1 of the Administration of the President of the \\ Russian Federation, Moscow, Russia; ' $S$ State Budget Institution of Higher Professional Education, The First Moscow Medical \\ University named after I.M. Sechenov of the Ministry of Healthcare, Moscow, Russia; ${ }^{f}$ State Budgeted Institution of Healthcare, \\ Stavropol Regional Clinical Hospital, Stavropol, Russia; ${ }^{9}$ Research Center of Otorhinolaryngology of the Federal Biomedical \\ Agency, Moscow, Russia
}

\section{Keywords}

Allergic rhinitis - Azelastine · Fluticasone propionate . Intranasal · MP-AzeFlu · Moderate-to-severe rhinitis · Russia · Seasonal

\begin{abstract}
Background: The novel intranasal formulation of azelastine hydrochloride (AZE) and fluticasone propionate (FP) in a single spray (MP-AzeFlu) was compared with a first-line intranasal antihistamine spray (AZE) in Russian seasonal allergic rhinitis (SAR) patients. Methods: Moderate-to-severe SAR/rhinoconjunctivitis patients ( $n=149$; aged $18-65$ years) were randomized to receive MP-AzeFlu (137/50 $\mu \mathrm{g}$ AZE/FP per spray) or AZE (137 $\mu \mathrm{g} / \mathrm{spray})$, both as 1 spray/nostril twice daily, in a multicenter, open-label, 14-day, parallel-group trial. The primary outcome was change from baseline in morning and evening reflective total nasal symptom score (rTNSS). Secondary end points included: change from baseline in reflective total ocular symptom score (rTOSS), reflective total
\end{abstract}

\begin{tabular}{ll}
\hline KARGER & $\begin{array}{l}\text { (c) } 2019 \text { The Author(s) } \\
\text { Published by S. Karger AG, Basel }\end{array}$ \\
E-Mail karger@karger.com & $\begin{array}{l}\text { This article is licensed under the Creative Commons Attribution- } \\
\text { NonCommercial-NoDerivatives 4.0 International License (CC BY- } \\
\text { NC-ND) (http://www.karger.com/Services/OpenAccessLicense). } \\
\text { Usage and distribution for commercial purposes as well as any dis- } \\
\text { tribution of modified material requires written permission. }\end{array}$
\end{tabular}

of 7 symptom scores (rT7SS), 28-item Rhinoconjunctivitis Quality of Life Questionnaire (RQLQ) overall score, and EuroQol-5D (EQ-5D) questionnaire score. Results: When compared with AZE-treated patients, those treated with MP-AzeFlu experienced significantly greater reductions in rTNSS (difference: $-2.47 ; 95 \%$ confidence interval $[\mathrm{Cl}]-3.65$ to $-1.30 ; p<0.001$ ), rTOSS (difference: $-1.62 ; 95 \% \mathrm{Cl}-2.32$ to $-0.92 ; p<0.001$ ), and rT7SS (difference: $-4.34 ; 95 \% \mathrm{Cl}-5.98$ to $-2.70 ; p<0.001$ ). Superior relief observed on day 2 with MP-AzeFlu versus AZE was sustained throughout the study. MP-AzeFlu-treated patients experienced a greater improvement in QoL than AZE-treated patients as measured by overall RQLQ score (mean \pm SD $2.91 \pm 1.08$ vs. $2.05 \pm 1.15$ ) and EQ-5D score (mean \pm SD $87.4 \pm 10.3$ vs. $83.0 \pm 12.8$ ). MP-AzeFlu was well tolerated. Conclusions: MP-AzeFlu was superior to AZE in reducing moderate-to-severe SAR symptoms, providing earlier and more complete symptom relief.

(c) 2019 The Author(s)

Published by S. Karger AG, Basel

Edited by: D.Y. Wang, Singapore.
Prof. Natalya I. Ilyina

NRC Institute of Immunology FMBA of Russia

Kashirskoye sh. 24, Building 2

RU-115478 Moscow (Russian Federation)

E-Mail instimmun@yandex.ru 


\section{Introduction}

Allergic rhinitis (AR) is a global health problem affecting as many as 500 million people worldwide [1]. A recent epidemiological study in 12 regions of the Russian Federation found AR symptoms in $18 \%$ of the population, among whom only $6.5 \%$ had a prior diagnosis of AR [2]. From the perspective of patients, AR causes significant morbidity, has a considerable impact on quality of life (QoL) $[3,4]$, and affects school and work attendance and productivity $[5,6]$. The symptoms of AR and allergic rhinoconjunctivitis in Russian patients are found to impair health-related QoL to the same extent as patients in westernized countries, by adversely impacting sleep, daily activities, physical and mental status, and social functioning [7]. AR results in considerable healthcare costs [1] and, if untreated, may have considerable economic and QoL implications [8-10]. The impact of AR on productivity is higher than that of asthma, diabetes, or heart disease $[9,10]$.

Intranasal antihistamines are considered the first-line therapy for AR in both the USA and Russia $[1,2]$. Currently, intranasal corticosteroids (INS) are the first-line treatment for AR according to the US Allergic Rhinitis and its Impact on Asthma (ARIA) guidelines [1]. INS are recommended for use in patients with mild, mild-tomoderate, and moderate-to-severe disease [1, 11-13]. However, current treatments for AR provide insufficient control for many patients. In both clinical trials and reallife studies, many patients experience inadequate symptom relief while on INS due to their slow onset of action, symptom breakthrough [14], and an efficacy ceiling of a $60 \%$ reduction from baseline in reflective total nasal symptom score (rTNSS) [15]. This leads to patient dissatisfaction and poor compliance with INS therapy [16].

Switching INS type is not an effective solution, as different types have comparable efficacy $[17,18]$. Consequently, many patients use multiple therapies [19] while searching for better and faster symptom relief [19], or simply "something that works" [20]. However, such multipharmacotherapy is not evidence-based [21-23] and therefore is not recommended by the guidelines $[1,13]$.

MP-AzeFlu (Dymista ${ }^{\circledR}$, Mylan Inc., Canonsburg, PA, USA) is a novel intranasal formulation of azelastine hydrochloride (AZE) and fluticasone propionate (FP) delivered as a single spray, i.e., comprising 2 active ingredients from 2 different medication classes (INS and intranasal antihistamines) with complementary effects. MP-AzeFlu encompasses all of the treatment principles in ARIA [1, 13] (i.e., antihistamine, antileukotriene, mast-cell stabilizing, and anti-inflammatory) in a single puff [24]. More- over, the efficacy and safety of MP-AzeFlu has been established in pivotal randomized controlled trials in the USA $[15,25]$, an open-label study in India $[26,27]$, and in real-life noninterventional studies conducted in Germany [28], the UK [28], Sweden [29], Norway [30], Denmark [31], and Romania [32].

MP-AzeFlu has previously been described as the drug of choice for the treatment of AR and a major breakthrough in the treatment of this disease [33]. The objective of this study was to assess the efficacy and safety of MP-AzeFlu in comparison with an AZE metered-dose nasal spray in a population of Russian seasonal AR (SAR)/ rhinoconjunctivitis patients.

\section{Material and Methods}

This was a multicenter, randomized, open-label, parallelgroup, phase 3 clinical study, carried out at 7 investigational sites in Russia between 25 April and 26 July 2014. The protocol and other study materials were approved by the Ministry of Healthcare of the Russian Federation and local ethics committees from the study centers, and the study was conducted in accordance with good clinical practice. Informed consent was obtained prior to the initiation of any study-related procedure.

\section{Participants}

Subjects aged 18-65 years, with a minimum 2-year history of moderate-to-severe SAR or rhinoconjuctivitis, current clinical rhinitis symptomatology, and a positive skin prick test to tree and grass pollens within the last year, were randomized. All subjects met ARIA criteria for moderate-to-severe SAR and had at least 1 of the following complaints: sleep disturbance, impairment of daily leisure and/or sports activities, impairment of study or work abilities, and bothersome symptoms. In addition, all subjects had to have an rTNSS of at least 8/12, with a congestion score of 2 or 3 during screening (day -3$)$. At the start of treatment, subjects were required to have a 12-h nasal congestion score of 2 or 3 measured by a minimum of 2 separate symptom assessments (morning or evening [a.m. or p.m.]) during the washout period, with at least 1 of the 2 assessments recorded within 2 days of day 1 (which could include the a.m. assessment on day 1).

Exclusion criteria included the presence of nasal mucosa ulcerations or nasal septum perforation, nasal or sinus surgery or episodes of acute rhinosinusitis within the last year, chronic rhinosinusitis or nasal polyposis, hypersensitivity to antihistamines and INS, bronchial asthma (mild intermittent allowed), immunotherapy within the last 6 months, and certain previous medications within a prespecified period (e.g., INS within the last 14 days or a systemic corticosteroid within the last 30 days).

\section{Planned Interventions and Timing}

The study comprised a 3-day washout period and a 14-day treatment period, with study visits at randomization (day 1), on day 8 , and at the end of the trial (day 14). On day 1, eligible patients were randomized in a 1:1 ratio to 14 days of treatment with either MP-AzeFlu nasal spray (137/50 $\mu \mathrm{g}$ AZE/FP per spray) or AZE (137 
Fig. 1. Patient disposition. MP-AzeFlu, a novel intranasal formulation of azelastine hydrochloride and fluticasone propionate in a single spray.

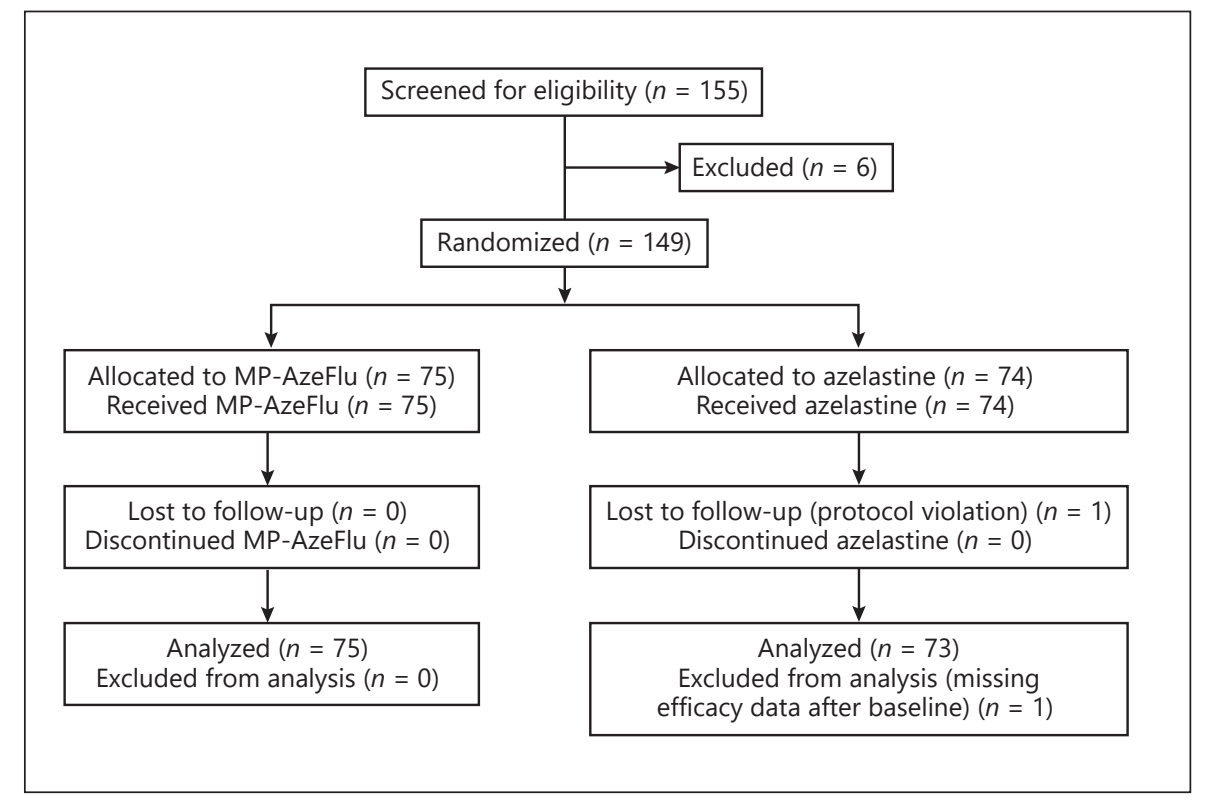

$\mu \mathrm{g} /$ spray), both administered as 1 spray/nostril twice daily, separated by approximately $12 \mathrm{~h}$ (total daily dose: AZE, $548 \mu \mathrm{g}$; FP [MP-AzeFlu only], $200 \mu \mathrm{g}$ ). Patients recorded nasal and ocular symptom scores twice daily in a diary. On day 1 , the a.m. assessment was before the treatment (included in baseline) and p.m. assessment upon treatment.

\section{Efficacy End Points}

The primary efficacy end point was the average change from baseline in combined (a.m. + p.m.) 12-h rTNSS for 13 days (days $2-14$ ) of the 14-day treatment period. Secondary end points included average change from baseline for this 13-day period in rTOSS, rT7SS (rT7SS = rTNSS + rTOSS), reflective individual nasal (congestion, itching, rhinorrhea, and sneezing) and ocular (itching, redness, and watering) symptom scores, daily change from baseline in 12-h (a.m. + p.m.) rTNSS, rTOSS, and rT7SS, time to $50 \%$ response, time to a near-to-complete resolution of symptoms (time to achieve a score of $\leq 1$ for each individual nasal symptom), an overall score for the 28-item Rhinoconjunctivitis Quality of Life Questionnaire (RQLQ), and a visual analogue scale (VAS) score of health status taken from the EuroQol-5D (EQ-5D) questionnaire. The scoring systems for rTNSS and rTOSS are described in the online supplementary data (for all online suppl. material, see www.karger.com/doi/10.1159/000494507).

\section{Safety End Points}

Safety end points included the incidence, intensity, and description of all adverse events (AEs) throughout the study, a focused nasal examination, and vital signs (blood pressure and heart rate).

\section{Statistical Analyses}

Change from baseline in rTNSS, rTOSS, rT7SS, and each individual nasal and ocular symptom for each treatment over time as well as treatment comparisons were assessed using baseline-adjusted analyses of variance.

Efficacy of MP-AzeFlu for the Treatment of SAR in Russia
Time to response was analyzed by Kaplan-Meier estimates and log-rank tests. Response was defined as change from baseline in a.m. + p.m. rTNSS of at least $50 \%$ and $\leq 1$ point remaining in each symptom score. A $\geq 50 \%$ rTNSS response was considered a substantial response, halving the patient's nasal symptom burden. The $\leq 1$-point score remaining for each symptom score of the rTNSS ensured complete/near-to-complete symptom relief from all nasal symptoms. This criterion is extremely strict, as it required each nasal symptom to be categorized as no more than mild severity, meaning that patients would effectively feel symptom-free. RQLQ and EQ-5D VAS results were summarized descriptively.

\section{Results}

\section{Patients}

In total, 155 patients were screened, 149 of whom were enrolled, randomized, and treated (Fig. 1). The study population consisted of these 149 patients, 75 of whom were allocated to receive MP-AzeFlu and 74 to receive AZE. One AZE-allocated patient dropped out due to "protocol violation including noncompliance," as their diary was not complete. The intention-to-treat population comprised the 148 study completers.

Treatment duration was a minimum of 11 days, with a mean (standard deviation [SD]) of $14.4( \pm 1.1)$ days in the MP-AzeFlu group and $14.1( \pm 1.0)$ days in the AZE group.

Patients in both treatment groups were comparable with regard to their baseline characteristics as presented in Table 1. The moderate-to-severe disease classification 


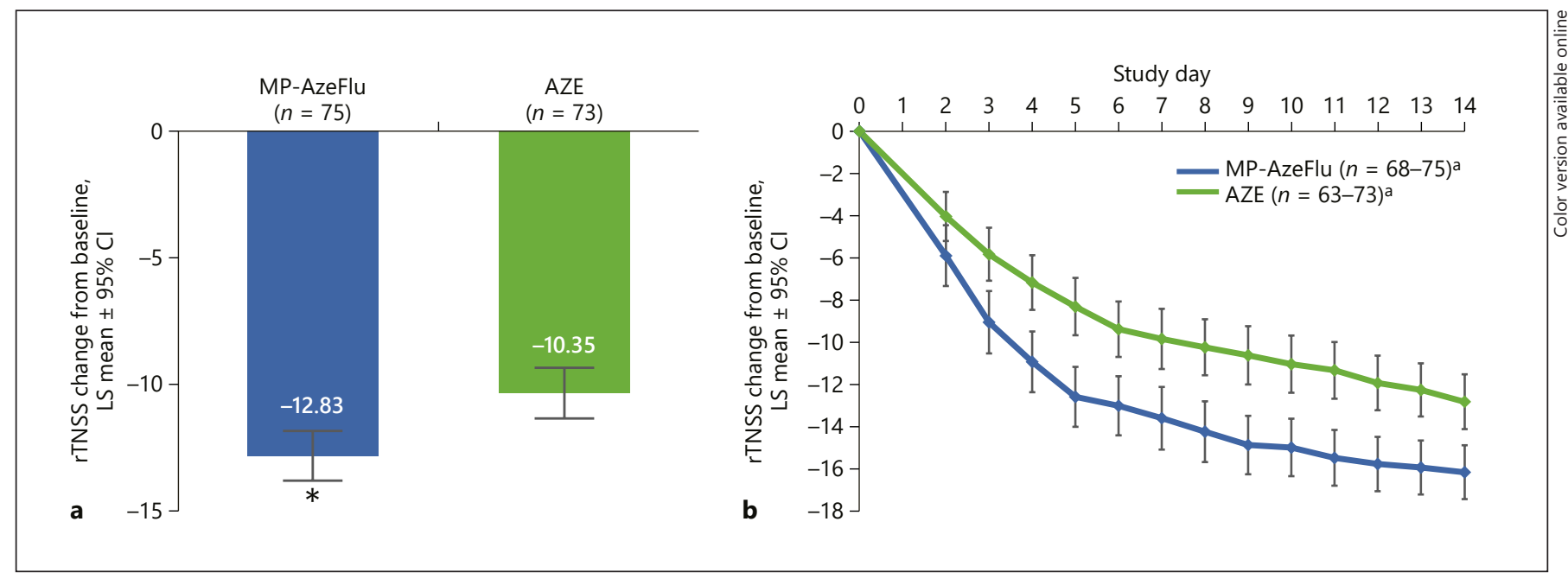

Fig. 2. a Average change of combined (a.m. + p.m.) 12-h reflective total nasal symptom score (rTNSS) during 14 days of treatment compared with baseline. b Daily change from baseline in combined rTNSS. AZE, azelastine hydrochloride; CI, confidence inter- val; LS, least squares; MP-AzeFlu, novel intranasal formulation of AZE and fluticasone propionate in a single spray. ${ }^{a}$ rTNSS (a.m. + p.m.) data may not have been available for all patients at all time points. ${ }^{*} p<0.001$.

Table 1. Baseline characteristics of study participants

\begin{tabular}{lcc}
\hline & $\begin{array}{l}\text { MP-AzeFlu } \\
(n=75)\end{array}$ & $\begin{array}{l}\text { AZE } \\
(n=73)\end{array}$ \\
\hline Mean age, years $( \pm$ SD) & $37.0( \pm 12.0)$ & $34.9( \pm 10.0)$ \\
Male gender, $n$ & $40(53.3 \%)$ & $31(42.5 \%)$ \\
Caucasian, $n$ & $75(100 \%)$ & $73(100 \%)$ \\
Mean height, cm $( \pm$ SD) & $172.8( \pm 9.6)$ & $171.2( \pm 9.2)$ \\
Mean weight, kg $( \pm$ SD) & $76.4( \pm 13.9)$ & $72.4( \pm 15.7)$ \\
Periods of disease manifestation, $n$ & & \\
$\quad$ Spring & $57(76.0 \%)$ & $46(63.0 \%)$ \\
$\quad$ Summer & $52(69.3 \%)$ & $51(69.9 \%)$ \\
$\quad$ Autumn & $13(17.3 \%)$ & $7(9.6 \%)$ \\
$\quad$ Winter & $2(2.7 \%)$ & $0(0 \%)$ \\
Mean $( \pm$ SD) rTNSS (a.m. + p.m.) & $19.9( \pm 2.2)$ & $8.1( \pm 5.0)$ \\
Mean $( \pm$ SD) rTOSS (a.m. + p.m.) & $9.2( \pm 5.1)$ & $3.1( \pm 1.1)$ \\
Mean $( \pm$ SD) overall RQLQ score & $3.5( \pm 1.1)$ & $7.0( \pm 6.5)$ \\
Mean $( \pm$ SD) time since diagnosis, years & $7.8( \pm 6.7)$ & $17 / 74(23.0 \%)^{\mathrm{a}}$ \\
Patients previously treated within last 30 days, $n$ & $16(21.3 \%)$ &
\end{tabular}

AZE, azelastine hydrochloride; MP-AzeFlu, novel intranasal formulation of AZE and fluticasone propionate in a single device; RQLQ, Rhinoconjunctivitis Quality of Life Questionnaire; rTNSS, reflective total nasal symptom score; rTOSS, reflective total ocular symptom score; SD, standard deviation.

${ }^{\mathrm{a}} n=74$.

was confirmed by ARIA criteria as well as by high baseline rTNSS, rTOSS, and overall RQLQ scores. Polysensitization was evident, with up to 11 sensitizing allergens reported per patient, though patients were most commonly allergic to 4 allergens $(n=32 ; 22 \%)$. The most frequent sensitizing allergens were cock's-foot $(n=83 ; 56 \%)$, Timothy grass $(n=81 ; 55 \%)$, fescue $(n=69 ; 47 \%)$, and a mixture of herb pollens $(n=66 ; 45 \%)$. 


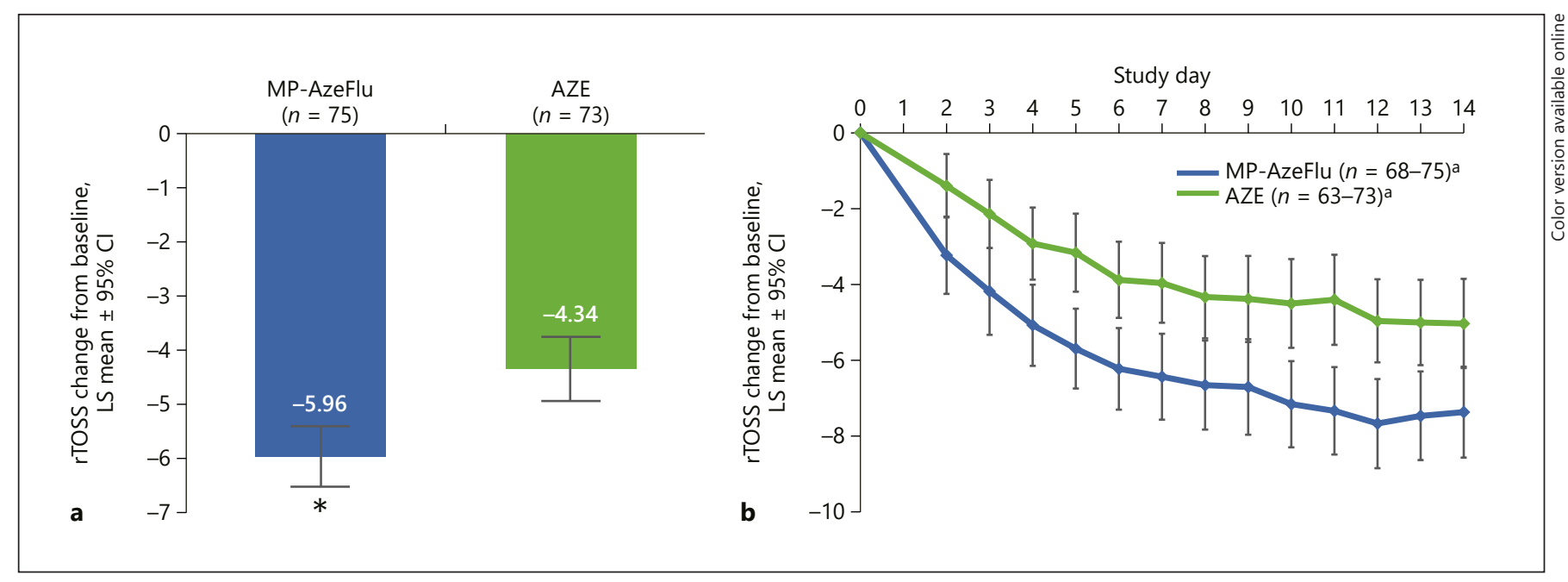

Fig. 3. a Average change of combined (a.m. + p.m.) 12-h reflective total ocular symptom score (rTOSS) during 14 days of treatment compared with baseline. b Daily change from baseline in combined rTOSS. AZE, azelastine hydrochloride; CI, confidence inter-

val; LS, least squares; MP-AzeFlu, novel intranasal formulation of AZE and fluticasone propionate in a single spray. ${ }^{\text {a }}$ rTOSS (a.m. + p.m.) data may not have been available for all patients at all time points. ${ }^{*} p<0.001$.

Table 2. Mean change from baseline during 14 days of treatment in individual nasal and ocular symptom scores

\begin{tabular}{|c|c|c|c|c|c|c|c|}
\hline & \multicolumn{2}{|c|}{ MP-AzeFlu $(n=75)$} & \multicolumn{2}{|c|}{$\mathrm{AZE}(n=73)$} & \multirow[t]{2}{*}{ Difference } & \multirow[t]{2}{*}{$95 \% \mathrm{CI}$} & \multirow[t]{2}{*}{$p$ value } \\
\hline & baseline & $\begin{array}{l}\text { change from } \\
\text { baseline }\end{array}$ & baseline & $\begin{array}{l}\text { change from } \\
\text { baseline }\end{array}$ & & & \\
\hline \multicolumn{8}{|c|}{ Individual nasal symptoms } \\
\hline Rhinorrhea & 4.58 & -2.91 & 4.44 & -2.20 & -0.71 & -1.04 to -0.39 & $<0.001$ \\
\hline Congestion & 5.80 & -3.59 & 5.76 & -2.83 & -0.76 & -1.11 to -0.41 & $<0.001$ \\
\hline \multicolumn{8}{|c|}{ Individual ocular symptoms } \\
\hline Itching & 3.38 & -2.20 & 3.04 & -1.62 & -0.58 & -0.85 to -0.31 & $<0.001$ \\
\hline Redness & 3.12 & -2.03 & 2.68 & -1.45 & -0.58 & -0.83 to -0.33 & $<0.001$ \\
\hline Watering & 2.72 & -1.76 & 2.36 & -1.35 & -0.41 & -0.66 to -0.15 & 0.002 \\
\hline
\end{tabular}

AZE, azelastine hydrochloride; CI, confidence interval; MP-AzeFlu, novel intranasal formulation of AZE and fluticasone propionate in a single device.

\section{Outcomes}

Primary Efficacy End Point

Baseline rTNSS (a.m. + p.m.) was $19.88 \pm 2.24$ and $19.30 \pm 2.30$ in the MP-AzeFlu and AZE groups, respectively. Patients treated with MP-AzeFlu experienced significantly better relief from their overall nasal symptoms. The least-squares (LS) mean reduction in rTNSS (a.m. + p.m.) across 13 days of treatment (days 2-14) was 12.83 for MP-AzeFlu-treated patients and 10.35 for AZE-treated patients (difference: -2.47 ; 95\% confidence interval [CI] -3.65 to $-1.30 ; p<0.001$ ) (Fig. 2a).
The superiority of MP-AzeFlu over AZE was observed on day 2 and was sustained to day 14 (Fig. 2b). In addition, significantly better relief was observed for each individual nasal symptom with MP-AzeFlu than with AZE $(p<$ 0.001) (Table 2).

\section{Secondary Efficacy End Points}

Baseline rTOSS (a.m. + p.m.) was $9.22 \pm 5.08$ in the MP-AzeFlu group and $8.07 \pm 5.03$ in the AZE group. Patients treated with MP-AzeFlu experienced significantly better relief from their overall ocular symptoms. MP- 


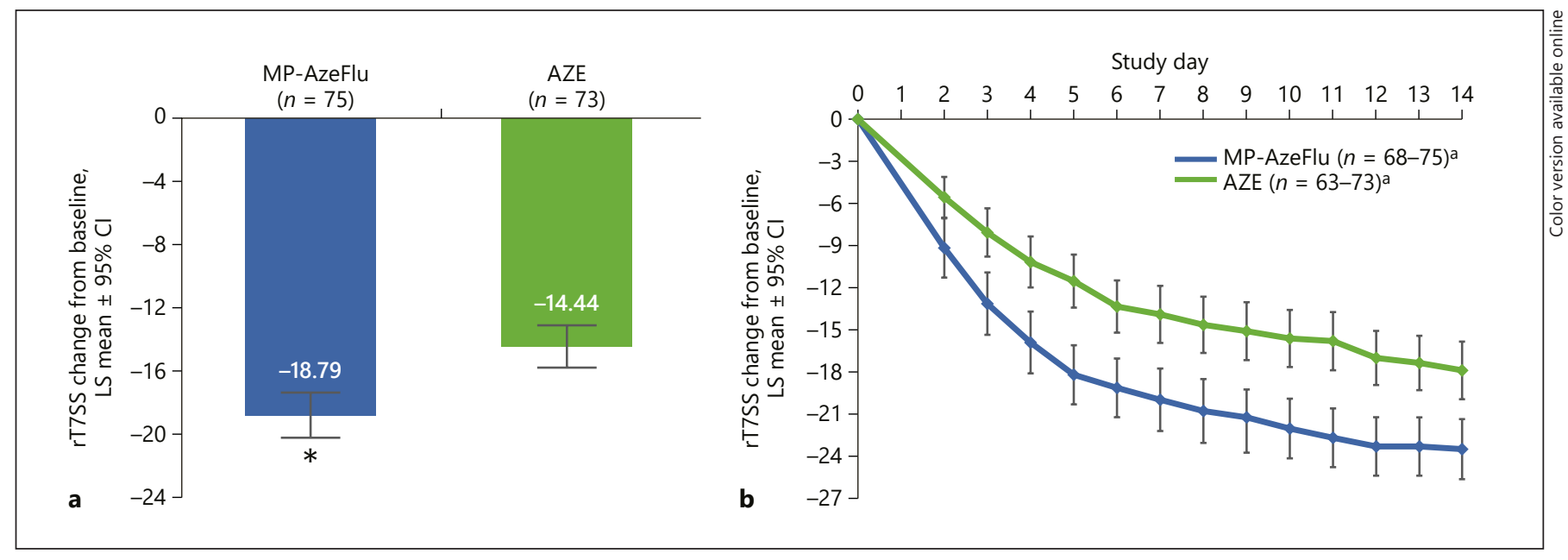

Fig. 4. a Average change of combined (a.m. + p.m.) 12-h reflective total of 7 symptom scores (rT7SS) during 14 days of treatment compared with baseline. b Daily change from baseline in combined rT7SS. AZE, azelastine hydrochloride; CI, confidence inter- val; LS, least squares; MP-AzeFlu, novel intranasal formulation of AZE and fluticasone propionate in a single spray. ${ }^{a}$ rT7SS (a.m. + p.m.) data may not have been available for all patients at all time points. ${ }^{*} p<0.001$.

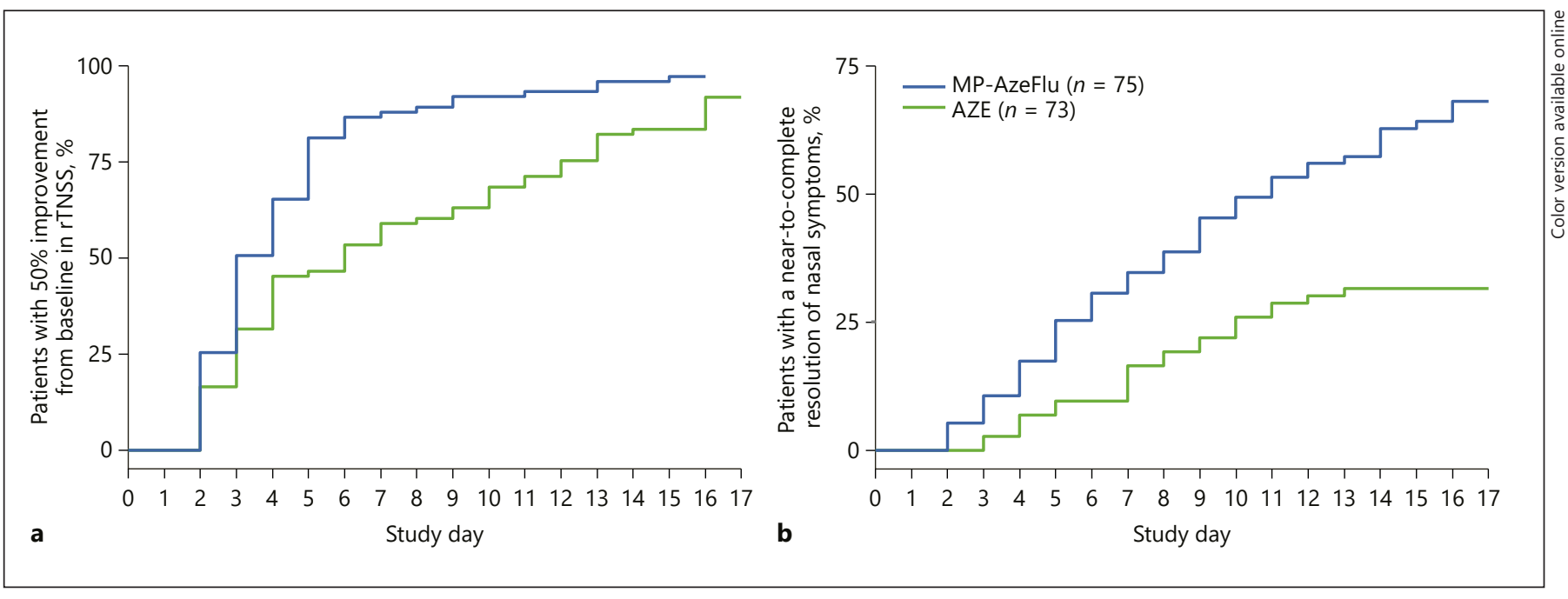

Fig. 5. a Percentage of patients exhibiting a 50\% improvement in rTNSS (a.m. + p.m.) by treatment day. b Percentage of patients with a near-to-complete resolution of nasal symptoms (score $\leq 1$ point for each nasal symptom) by treatment day. AZE, azelastine

AzeFlu-treated patients experienced a 5.96-point reduction in rTOSS (a.m. + p.m.) compared with the 4.34-point reduction in the AZE-treated group for days 2-14 of treatment (difference: -1.62 points; $95 \% \mathrm{CI}-2.32$ to $-0.92 ; p<0.001$ ) (Fig. 3a). The superiority of MP-AzeFlu over AZE was observed on day 2 of treatment and was sustained to day 14 (Fig. 3b). Significantly better relief with MP-AzeFlu was also observed for each individual ocular symptom $(p<0.01)$ (Table 2 ). hydrochloride; MP-AzeFlu, novel intranasal formulation of AZE and fluticasone propionate in a single spray; rTNSS, reflective total nasal symptom score.

Overall, MP-AzeFlu provided significantly better relief from the entire reflective rhinoconjunctivitis symptoms than AZE (18.79-point vs. 14.44-point reductions in rT7SS score [a.m. + p.m.], respectively; difference: -4.34 ; $95 \%$ CI -5.98 to $-2.70 ; p<0.001$ ) (Fig. $4 \mathrm{a}$ ). The superiority of MP-AzeFlu over AZE developed on day 2 of treatment and was sustained to day 14 (Fig. 4b).

Nearly all MP-AzeFlu-treated patients experienced at least a $50 \%$ reduction from baseline in their nasal symp- 


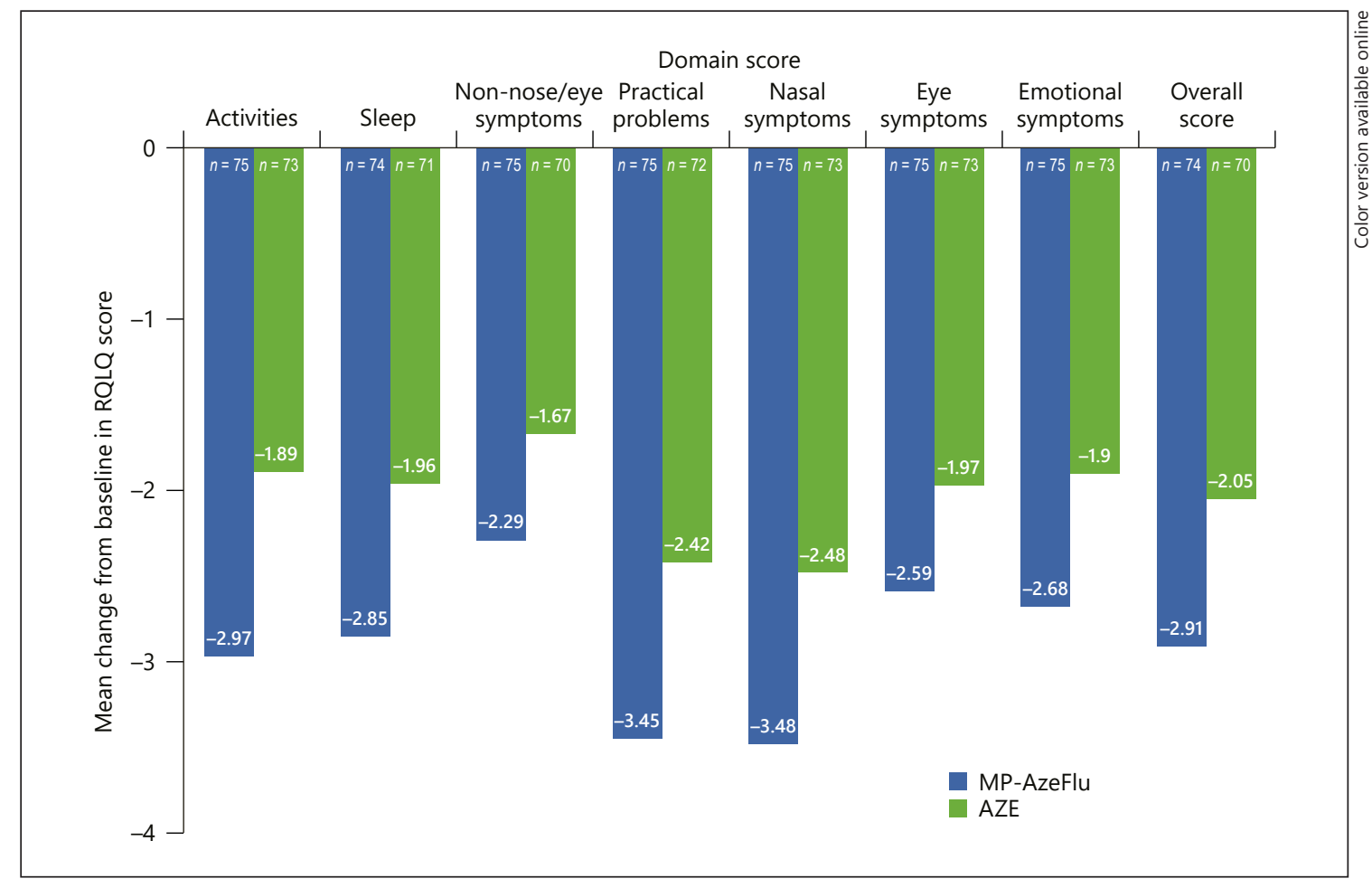

Fig. 6. Mean change from baseline in quality of life measured with the adult RQLQ for overall change and each of the 7 subscores for MP-AzeFlu versus AZE. AZE, azelastine hydrochloride; MP-AzeFlu, novel intranasal formulation of AZE and fluticasone propionate in a single spray; RQLQ, Rhinoconjunctivitis Quality of Life Questionnaire.

toms after 14 days of treatment, and they responded faster $(p<0.0001)$ than those treated with AZE (a median time to response of 3 and 6 days, respectively) (Fig. 5a). Approximately two-thirds of patients treated with MPAzeFlu achieved near-to-complete nasal symptom relief with MP-AzeFlu after 14 days of treatment compared with approximately one-third of AZE patients, and patients treated with MP-AzeFlu achieved this response up to 6 days faster than AZE-treated patients $(p<0.0001$; Fig. 5b).

\section{Quality of Life}

Patients treated with MP-AzeFlu experienced a greater improvement than patients treated with AZE in overall RQLQ score $(2.91 \pm 1.08$ and $2.05 \pm 1.15$, respectively) and each of the 7 individual domain scores (Fig. 6). The difference was clinically relevant [34]. Similar to RQLQ scores, EQ-5D VAS scores increased more for patients treated with MP-AzeFlu than for those treated with AZE. Scores increased from $61.0 \pm 16.6$ at baseline to $87.4 \pm$ 10.3 on day 14 in the MP-AzeFlu group and from $63.5 \pm$
17.1 to $83.0 \pm 12.8$ in the AZE group. The increase in VAS score from baseline to day 14 was 26.4 for the MP-AzeFlu group and 19.5 for the AZE group.

\section{Safety}

The proportion of subjects with an $\mathrm{AE}$ was similar in the 2 treatment groups: 11/75 (14.7\%) patients reported a total of 16 events in the MP-AzeFlu group and 11/74 (14.9\%) patients reported a total of 24 events in the AZE group. The most frequent $\mathrm{AE}$ was headache, reported by 5 patients in each treatment group (Table 3); headache was found to be unlikely related to treatment in 2 patients in the MP-AzeFlu group and 3 in the AZE group. All treatment-related AEs were mild-to-moderate in severity, and no serious AEs were recorded in the study.

No clinically significant changes in patients' vital signs or blood chemistry were observed throughout the study. Anterior rhinoscopy did not reveal any nasal mucosa damage throughout the study. 
Table 3. Most frequently reported adverse events

\begin{tabular}{lll}
\hline & $\begin{array}{l}\text { MP-AzeFlu, } n(\%) \\
(n=75)\end{array}$ & $\begin{array}{l}\text { AZE, } n(\%) \\
(n=74)\end{array}$ \\
\hline Headache & $5(6.7)$ & $5(6.8)$ \\
Dysgeusia & $1(1.3)$ & $3(4.1)$ \\
Somnolence & $2(2.7)$ & $1(1.4)$ \\
Sneezing & $1(1.3)$ & $2(2.7)$ \\
\hline
\end{tabular}

Adverse events were reported in at least 3 patients. AZE, azelastine hydrochloride; MP-AzeFlu, novel intranasal formulation of AZE and fluticasone propionate in a single device.

\section{Discussion}

The results of this study provide, for the first time, important evidence of the efficacy of MP-AzeFlu in Russian patients with moderate-to-severe SAR or rhinoconjunctivitis. The efficacy of MP-AzeFlu has been established using traditional measures as well as newly defined clinically relevant ones (as recommended by the European Medicines Agency). The superiority of MP-AzeFlu over AZE was apparent in both nasal and ocular symptom relief, the clinically relevant response, and the QoL, with both treatments equally well tolerated.

MP-AzeFlu was shown to be significantly more effective than first-line treatment with an antihistamine (AZE) for SAR. In our study, the efficacy of MP-AzeFlu was assessed in a clinically relevant way, i.e., in a way that means something to patients and physicians, and answers the question "How much better will I feel and how quickly will I get better?" Almost all patients treated with MPAzeFlu in this study experienced a clinically relevant regress of their nasal symptom burden in 2 weeks. After just 5 days, more than three-quarters of the patients developed a $50 \%$ response to MP-AzeFlu.

Assuming similar results across practices, physicians treating AR could see more than twice as many MP-AzeFlutreated patients than AZE-treated patients experiencing complete/near-to-complete symptom relief, and many days sooner. This is important because the time to achieve significant symptom reduction is crucial for patients with $\mathrm{AR}$ and is a trigger for maintaining patient compliance [20].

The findings of this study are consistent with those of previous randomized controlled trials of MP-AzeFlu carried out in the USA $[15,25]$. Data from US placebo-controlled studies show that MP-AzeFlu provided twice the overall nasal and ocular symptom relief than either FP or AZE monotherapy. Point estimates for treatment differenc- es in the Russian study were even higher than in the US studies, confirming the high efficacy of MP-AzeFlu. As in this study, more US MP-AzeFlu-treated patients achieved a $50 \%$ reduction from baseline in rTNSS and complete/ near-to-complete symptom relief (and this occurred about a week faster) than those treated with commercially available active comparators intranasal FP or AZE [15]. MPAzeFlu also provided greater symptom relief, irrespective of disease severity, most effectively treating the entire spectrum of nasal and ocular symptoms, and targeted patients' most bothersome symptoms [15]. Of note, MP-AzeFlu was 3 times more effective than FP for congestion [15].

MP-AzeFlu also demonstrated a consistent response across seasons and large geographic regions (i.e., Europe, the USA, and Asia) [25]. In real-life studies, MP-AzeFlu was found to provide effective and rapid symptom control in all age groups (adults and adolescents) of patients with SAR or perennial AR, and in those with more or less severe disease, with responder rates higher than those observed in controlled clinical trials [28].

The open-label study design remains the main limitation of this study. The generalizability of these data is an inherent limitation of all randomized trials [35] since inclusion and exclusion criteria have to be in agreement with the US FDA and European Medicines Agency guidelines.

In conclusion, MP-AzeFlu provided significantly better, more rapid, and more complete symptom control than AZE in a Russian cohort with moderate-to-severe SAR or rhinoconjunctivitis, supporting its position as an effective treatment for these patients.

\section{Acknowledgements}

Medical writing assistance in the preparation of this manuscript was provided by Patrick Harty, PhD, Morgan Hill, PhD, and Roger Hill, PhD, and editorial support by Paula Stuckart (all from Ashfield Healthcare Communications, Middletown, CT, USA), and funded by MEDA Pharma GmbH \& Co. KG. This study was funded by MEDA, a Mylan company.

\section{References}

1 Bousquet J, Khaltaev N, Cruz AA, Denburg J, Fokkens WJ, Togias A, et al; AllerGen. Allergic Rhinitis and its Impact on Asthma (ARIA) 2008 update (in collaboration with the World Health Organization, GA(2)LEN and AllerGen). Allergy. 2008 Apr;63 Suppl 86:8-160.

2 Chuchalin AG, Khaltaev N, Antonov NS, Galkin DV, Manakov LG, Antonini P, et al. Chronic respiratory diseases and risk factors in 12 regions of the Russian Federation. Int J Chron Obstruct Pulmon Dis. 2014 Sep;9:96374 . 
3 Bousquet PJ, Demoly P, Devillier P, Mesbah $\mathrm{K}$, Bousquet J. Impact of allergic rhinitis symptoms on quality of life in primary care. Int Arch Allergy Immunol. 2013;160(4):393400.

4 Small M, Piercy J, Demoly P, Marsden H. Burden of illness and quality of life in patients being treated for seasonal allergic rhinitis: a cohort survey. Clin Transl Allergy. 2013 Oct; $3(1): 33$.

5 Walker S, Khan-Wasti S, Fletcher M, Cullinan P, Harris J, Sheikh A. Seasonal allergic rhinitis is associated with a detrimental effect on examination performance in United Kingdom teenagers: case-control study. J Allergy Clin Immunol. 2007 Aug;120(2):381-7.

6 Szeinbach SL, Seoane-Vazquez EC, Beyer A, Williams PB. The impact of allergic rhinitis on work productivity. Prim Care Respir J. 2007 Apr;16(2):98-105.

7 Maspero J, Lee BW, Katelaris CH, Potter PC, Cingi C, Lopatin A, et al. Quality of life and control of allergic rhinitis in patients from regions beyond western Europe and the United States. Clin Exp Allergy. 2012 Dec;42(12): 1684-96.

8 Schoenwetter WF, Dupclay L Jr, Appajosyula S, Botteman MF, Pashos CL. Economic impact and quality-of-life burden of allergic rhinitis. Curr Med Res Opin. 2004 Mar;20(3): 305-17.

9 Lamb CE, Ratner PH, Johnson CE, Ambegaonkar AJ, Joshi AV, Day D, et al. Economic impact of workplace productivity losses due to allergic rhinitis compared with select medical conditions in the United States from an employer perspective. Curr Med Res Opin. 2006 Jun;22(6):1203-10.

10 de la Hoz Caballer B, Rodríguez M, Fraj J, Cerecedo I, Antolín-Amérigo D, Colás C. Allergic rhinitis and its impact on work productivity in primary care practice and a comparison with other common diseases: the Cross-sectional study to evAluate work Productivity in allergic Rhinitis compared with other common dIseases (CAPRI) study. Am J Rhinol Allergy. 2012 Sep-Oct;26(5):390-4.

11 Wallace DV, Dykewicz MS, Bernstein DI, Blessing-Moore J, Cox L, Khan DA, et al.; Joint Task Force on Practice; American Academy of Allergy; Asthma \& Immunology; American College of Allergy; Asthma and Immunology; Joint Council of Allergy, Asthma and Immunology. The diagnosis and management of rhinitis: an updated practice parameter. J Allergy Clin Immunol. 2008 Aug; 122(2 Suppl):S1-84.

12 Scadding GK, Durham SR, Mirakian R, Jones NS, Leech SC, Farooque S, et al.; British Society for Allergy and Clinical Immunology. BSACI guidelines for the management of allergic and non-allergic rhinitis. Clin Exp Allergy. 2008 Jan;38(1):19-42.

13 Brozek JL, Bousquet J, Baena-Cagnani CE, Bonini S, Canonica GW, Casale TB, et al.;
Global Allergy and Asthma European Network; Grading of Recommendations Assessment, Development and Evaluation Working Group. Allergic Rhinitis and its Impact on Asthma (ARIA) guidelines: 2010 revision. J Allergy Clin Immunol. 2010 Sep;126(3):46676.

14 Schatz M. A survey of the burden of allergic rhinitis in the USA. Allergy. 2007;62(Suppl 85):9-16.

15 Meltzer E, Ratner P, Bachert C, Carr W, Berger W, Canonica GW, et al. Clinically relevant effect of a new intranasal therapy (MP29-02) in allergic rhinitis assessed by responder analysis. Int Arch Allergy Immunol. 2013;161(4): 369-77.

16 Ciprandi G, Incorvaia C, Scurati S, Puccinelli P, Soffia S, Frati F, et al. Patient-related factors in rhinitis and asthma: the satisfaction with allergy treatment survey. Curr Med Res Opin. 2011 May;27(5):1005-11.

17 Mandl M, Nolop K, Lutsky BN. Comparison of once daily mometasone furoate (Nasonex) and fluticasone propionate aqueous nasal sprays for the treatment of perennial rhinitis. 194-079 Study Group. Ann Allergy Asthma Immunol. 1997 Oct;79(4):370-8.

18 Okubo K, Nakashima M, Miyake N, Komatsubara M, Okuda M. Comparison of fluticasone furoate and fluticasone propionate for the treatment of Japanese cedar pollinosis. Allergy Asthma Proc. 2009 Jan-Feb;30(1):8494.

19 Price D, Scadding G, Ryan D, Bachert C, Canonica GW, Mullol J, et al. The hidden burden of adult allergic rhinitis: UK healthcare resource utilisation survey. Clin Transl Allergy. 2015 Nov;5(5):39.

20 Marple BF, Fornadley JA, Patel AA, Fineman SM, Fromer L, Krouse JH, et al.; American Academy of Otolaryngic Allergy Working Group on Allergic Rhinitis. Keys to successful management of patients with allergic rhinitis: focus on patient confidence, compliance, and satisfaction. Otolaryngol Head Neck Surg. 2007 Jun;136(6 Suppl):S107-24.

21 Anolik R; Mometasone Furoate Nasal Spray with Loratadine Study Group. Clinical benefits of combination treatment with mometasone furoate nasal spray and loratadine vs monotherapy with mometasone furoate in the treatment of seasonal allergic rhinitis. Ann Allergy Asthma Immunol. 2008 Mar; 100(3):264-71.

22 Di Lorenzo G, Pacor ML, Pellitteri ME, Morici G, Di Gregoli A, Lo Bianco C, et al. Randomized placebo-controlled trial comparing fluticasone aqueous nasal spray in mono-therapy, fluticasone plus cetirizine, fluticasone plus montelukast and cetirizine plus montelukast for seasonal allergic rhinitis. Clin Exp Allergy. 2004 Feb;34(2):25967.

23 Esteitie R, deTineo M, Naclerio RM, Baroody FM. Effect of the addition of montelukast to fluticasone propionate for the treatment of perennial allergic rhinitis. Ann Allergy Asthma Immunol. 2010 Aug;105(2):155-61.

24 Mösges R. All of ARIA in one puff? Int Arch Allergy Immunol. 2014;163(3):163-4.

25 Carr W, Bernstein J, Lieberman P, Meltzer E, Bachert C, Price D, et al. A novel intranasal therapy of azelastine with fluticasone for the treatment of allergic rhinitis. J Allergy Clin Immunol 2012;129(5):1282-9.e10.

26 Price D, Shah S, Bhatia S, Bachert C, Berger $\mathrm{W}$, Bousquet J, et al. A new therapy (MP2902) is effective for the long-term treatment of chronic rhinitis. J Investig Allergol Clin Immunol. 2013;23(7):495-503.

27 Berger WE, Shah S, Lieberman P, Hadley J, Price D, Munzel U, et al. Long-term, randomized safety study of MP29-02 (a novel intranasal formulation of azelastine hydrochloride and fluticasone propionate in an advanced delivery system) in subjects with chronic rhinitis. J Allergy Clin Immunol Pract 2014;2(2): 179-85.

28 Klimek L, Bachert C, Mösges R, Munzel U, Price D, Virchow JC, et al. Effectiveness of MP29-02 for the treatment of allergic rhinitis in real-life: results from a noninterventional study. Allergy Asthma Proc. 2015 Jan-Feb; 36(1):40-7.

29 Stjarne P, Strand V, Theman K, Kuhl H, Ehngage A. Real-life effectiveness of a new allergic rhinitis therapy (MP29-02) in Sweden. European Academy of Allergy and Clinical Immunology Congress; 2015 June 6-10; Barcelona.

30 Dollner R, Lorentz Larsen P, Sheyauldeen S, Kuhl H, Steinsvag S. Real-life effectiveness of a new allergic rhinitis therapy (MP29-02) in Norway. European Academy of Allergy and Clinical Immunology Congress; 2015 June 6-10; Barcelona.

31 Haahr P, Jacobsen C, Blegvad S, Christensen M, Kuhl H, Nielsen K. Real-life effectiveness of a new allergic rhinitis therapy (MP29-02) in Denmark. European Academy of Allergy and Clinical Immunology Congress; 2015 June 6-10; Barcelona.

32 Agache I, Doros IC, Leru PM, Bucur I, Poenaru M, Sarafoleanu C. MP-AzeFlu provides rapid and effective allergic rhinitis control: results of a non-interventional study in Romania. Rhinology. 2018 Mar;56(1):33-41.

33 Leung D, Szefler S. Associated Editors of the JACI. MP29-02: a major advancement in the treatment of allergic rhinitis. J Allergy Clin Immunol. 2012;129:1216.

34 Barnes ML, Vaidyanathan S, Williamson PA, Lipworth BJ. The minimal clinically important difference in allergic rhinitis. Clin Exp Allergy. $2010 \mathrm{Feb} ; 40(2): 242-50$.

35 Costa DJ, Amouyal M, Lambert P, Ryan D, Schunemann HJ, Daures JP, et al. How representative are clinical study patients with allergic rhinitis in primary care? J Allergy Clin Immunol. 2011;127(4):920-6.e1. 